\title{
Why Brazilian women suffer more from depression and suicidal ideation: a mediation analysis of the role of violence
}

\author{
Marina X. Carpena, ${ }^{1}$ (iD Francine dos S. Costa, ${ }^{1}$ (iD Thais Martins-Silva, ${ }^{1}$ (iD Mariana O. Xavier, ${ }^{1}$ \\ Christian Loret de Mola ${ }^{1,2,3}$ iD \\ ${ }^{1}$ Programa de Pós-Graduação em Epidemiologia, Departamento de Medicina Social (DMS), Universidade Federal de Pelotas (UFPel), \\ Pelotas, RS, Brazil. ${ }^{2}$ Programa de Pós-Graduação em Saúde Pública, Universidade Federal do Rio Grande (FURG), Rio Grande, RS, Brazil. \\ ${ }^{3}$ Universidad Cientifica del Sur, Lima, Peru.
}

\begin{abstract}
Objective: We aimed to investigate the mediating effect of violence by a family member or acquaintance on biological sex, depression and suicidal ideation.

Methods: We used data from the 2013 Brazilian National Health Survey, a cross-sectional nationwide survey. Major depressive episode and suicidal ideation were evaluated with the Patient Health Questionnaire. Violence victimization and other sociodemographic variables were self-reported. We used logistic regression to estimate the $\mathrm{OR}, 95 \% \mathrm{Cl}$ and $\mathrm{G}$-computation to calculate the natural direct and indirect effects.

Results: A total of 60,202 individuals were evaluated. Women had higher prevalences of major depressive episode $(\mathrm{OR}=2.36 ; 95 \% \mathrm{Cl} 2.03-2.74)$, suicidal ideation $(\mathrm{OR}=2.02 ; 95 \% \mathrm{Cl} 1.73-2.36)$ and violence victimization $(\mathrm{OR}=1.73 ; 95 \% \mathrm{Cl} 1.45-2.06)$. The mediation analysis showed that $10.6 \%$ of the association between sex and major depressive episode and $8.0 \%$ of the association between sex and suicidal ideation is explained by violence.

Conclusions: Women in Brazil have an increased risk of major depressive episode and suicidal ideation, and this association is mediated, in part, by the fact that they suffer more violence from family members or acquaintances.
\end{abstract}

Keywords: Depression; suicide; violence; sex; population-based study

\section{Introduction}

Depression and suicidal ideation are about two to three times higher in women than men. ${ }^{1-7}$ Theories explaining this higher risk focus on biological issues, despite the evident impact of social variables and inequities. Although scientists have reported this gap for many decades, empirical evidence explaining this difference is scarce. ${ }^{8-10}$

Violence is a global public health problem and women are at a higher risk of victimization, especially from family members or acquaintances. ${ }^{11-15}$ According to the United Nations' Sustainable Development Goals Report 2019, from 2005 to $2017,18 \%$ of women between 15 and 49 years of age had experienced physical and/or sexual violence by an intimate partner in the previous 12 months. The majority $(64 \%)$ of victims of intimate partner/family-related homicides in 2017 were also women. ${ }^{16}$

Violence may lead to death in the most extreme cases, and when it does not, it could have important consequences for the victim's mental health and quality of life, independently of the type of violence (physical, sexual, economic or psychological). ${ }^{17}$ Many studies have

Correspondence: Marina Xavier Carpena, Universidade Federal de Pelotas, Rua Mal. Deodoro, 1160, $2^{\circ}$ andar, CEP 96020-220, Pelotas, RS, Brazil.

E-mail: marinacarpena_@hotmail.com

Submitted Jun 10 2019, accepted Jan 02 2020, Epub Jun 012020. demonstrated the association between violence and mental illness, and that certain kinds violence, such as that by intimate partners or acquaintances, are more prevalent in women. Other studies have shown that violence might play different roles in depressive disorder in men and women in adulthood. ${ }^{12,13}$ Gender inequality and exposure to severe adversity, especially violence, might explain the higher prevalence of certain mental disorders in women. ${ }^{10,12,13}$

Nevertheless, little is known about the role of violence in the causal pathway of mental disorders in both sexes, and none have explored it using a counterfactual framework. Therefore, the aim of this study was to investigate the mediating effect of violence in the association between biological sex and depression or suicidal ideation in adult respondents to a nationwide Brazilian survey.

\section{Methods}

This study used data from the 2013 National Health Survey (Pesquisa Nacional de Saúde), a cross-sectional population-based survey. The National Health Survey

How to cite this article: Carpena MX, Costa FS, Martins-Silva T, Xavier MO, Loret de Mola C. Why Brazilian women suffer more from depression and suicidal ideation: a mediation analysis of the role of violence. Braz J Psychiatry. 2020;42:469-474. http://dx.doi. org/10.1590/1516-4446-2019-0572 
covered the entire territory of Brazil based on tracts used in the 2010 census. Areas with special characteristics or small populations were excluded.

The sampling process was carried out in three stages: census tracts (or set of sectors) were the primary sampling units, households were the second-stage units and residents aged 18 years or over were the third-stage units. The original study selected 69,954 households and involved $13.9 \%$ losses and refusals. Through a simple randomization process, one individual per household was selected and invited to participate in the face-to-face survey. Trained interviewers applied the survey using personal digital assistants. Written informed consent was obtained from all participants.

Based on the probability of sample participation, weighting was used to ensure national, regional, and state representativeness. Further details on the methodology, sampling and weighting factors can be found in the National Health Survey technical report. ${ }^{18}$

The outcomes considered in the present study were major depressive episode (MDE), determined through the Patient Health Questionnaire (PHQ-9), which identifies cases according to DSM-IV criteria, and suicidal ideation, which was determined with the final question of the PHQ9: "In the last two weeks, how many days did you think about hurting yourself in any way or think that it would be better to be dead?" Possible answers to the question were: None, Less than one week, One week or more, and Almost every day. If the respondent's answer was anything other than "none", he/she was considered positive for suicidal ideation. The PHQ-9 has been validated for adults in the general population of Brazil. ${ }^{19}$

We investigated whether respondents were victims of family violence (mediator/effect modifier) with the question: "In the last 12 months, did you experience any violence or aggression from an acquaintance (such as father, mother, child, spouse, partner, boyfriend, friend, neighbor)?" The variable biological sex (male and female) was our main exposure variable.

Sociodemographic data, collected through the general questionnaire, were used as covariables: age in complete years ( 18 to $24 ; 25$ to $34 ; 35$ to $44 ; 45$ to $54 ; 55$ to 64 ; 65 or more); self-reported skin color (white, black, brown, yellow or indigenous); education (none or incomplete elementary school; complete elementary school or incomplete secondary school; complete secondary school or incomplete higher education; complete higher education); and wealth index quintiles (WIQ). For WIQ classification, the respondents were asked about residential characteristics and household belongings, such as: house or apartment, number of bedrooms, having a domestic worker, and the number and type of home appliances (such as televisions). The five quintiles ranged from the poorest (1st) to the richest (5th).

Data analysis was performed in Stata version 14.0 (Stan Corp, College Station, Texas, USA), and the clustering effect was considered using the "survey" command. We calculated total and stratified prevalence of the variables and used chi-square tests for heterogeneity. Odds ratios (OR) and $95 \%$ confidence intervals $(95 \% \mathrm{Cl})$ were estimated with logistic regression to estimate the association between sex, MDE, and suicidal ideation. The analysis was stratified according to violence, and a multiplicative interaction term between both sexes and violence was included in the regression models to determine if there was a modifier effect. We also calculated the relative excess risk due to interaction, a measure similar to additive interaction that uses risk ratios or odds ratios rather than risks. ${ }^{20}$

To estimate the potential mediating effect of violence, we used the G-formula, a parametric computation that uses Monte Carlo simulations. The G-formula offers greater flexibility than traditional mediation methods, including modelling non-linearities and allowing for mediator-outcome confounding and exposure-mediator interaction. We calculated the total effect, the natural direct effect (NDE), the natural indirect effect (NIE), and the controlled direct effect (CDE).$^{21}$ The NDE represents the effect of exposure (being a woman) on the outcomes (MDE or suicidal ideation) that is not explained by the mediator (violence), while the NIE estimates the effect that is explained by it. The sum of the NDE and NIE represents the total effect, and the quotient of the NIE divided by the total effect represents the percentage of the effect explained by the mediator. On the other hand, the CDE represents the direct effect of exposure on the outcome if the mediator is controlled (maintaining it constant at one level independently of exposure). In our case we investigated what would happen if no one was exposed to violence, regardless of gender. We created and tested two different models, one for MDE and one for suicidal ideation, adjusting for age, skin color, education and WIQ (Figure 1). Education was included in the model as an intermediate confounder.

\section{Ethics statement}

All procedures in this study complied with the ethical standards of the relevant national and institutional committees on human experimentation and with the Helsinki Declaration. All procedures involving human participants were approved by the Brazilian Ministry of Health's National Commission of Ethics in Research for Human Beings (number 328.159).

\section{Results}

Of the 60,202 interviewed individuals, $52.9 \%$ were female, $21.6 \%$ were between 25 and 34 years of age, most selfreported their skin color as white $(47.5 \%)$, completed secondary school or had incomplete higher education (38.1\%), and belonged to the poorest quintile $(20.3 \%)$. Overall, $4.1 \%$ presented MDE (5.6\% in women vs. $2.5 \%$ in men) and $3.8 \%$ presented suicidal ideation (4.9\% in women vs. $2.5 \%$ in men) and $2.5 \%$ had suffered family violence in the last 12 months (3.1\% in women vs. $1.8 \%$ in men).

Older men and women with less education and a lower WIQ had a higher prevalence of MDE and suicidal ideation. Violence victimization was more frequent in poor young black women with low education (Table 1).

Table 2 shows that women's odds of MDE and suicidal ideation were 2.36 and 2.02 times higher than men, respectively. When the analysis was stratified according to violence victimization, female victims had a higher risk 

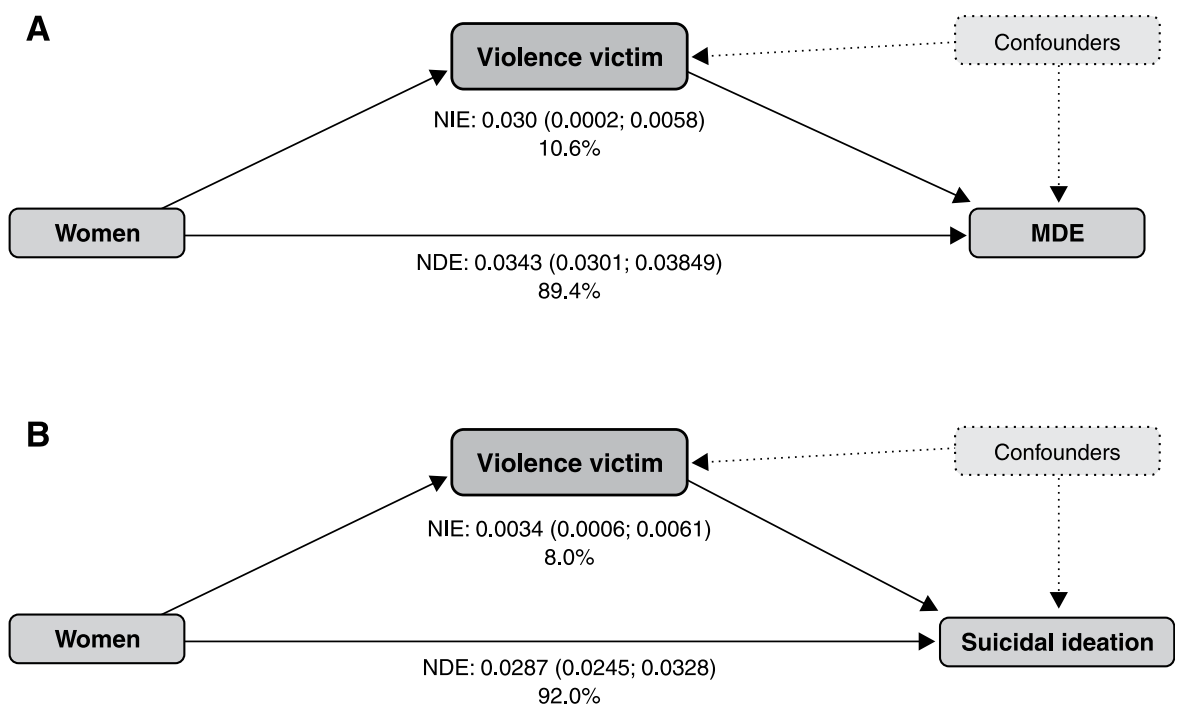

Figure 1 The mediating effect of violence in the association of being a woman and: (A) major depressive episode, (B) suicidal ideation. The main exposure was being a woman, with men as the reference group. As a mediator, violence was categorized as victimization vs. not having a major depressive episode vs. not having suicidal ideation. The natural direct and indirect effects were calculated with G-computation. Confounders included age, skin color, wealth index, and education (intermediate confounder). The percentages are based on the natural direct and indirect effects and represent the proportion of the association explained by each pathway. MDE = major depressive episode; NIE = natural indirect effect; NDE = natural direct effect.

of $\mathrm{MDE}(\mathrm{OR}=3.31 ; 95 \% \mathrm{Cl} 2.54-4.32)$ and suicidal ideation (OR = 3.85; 95\%Cl 2.83-5.25). However, confidence intervals from multiplicative interaction terms included the reference, suggesting that these differences could be due to chance (MDE interaction term: $1.37 ; 95 \% \mathrm{Cl}$ 0.86-2.19; suicidal ideation interaction term: $1.65 ; 95 \% \mathrm{Cl} 1.00-2.71)$.

When the additive interactions were calculated using the OR from our regression models, we found that the relative excess risk due to interaction for depression was $5.97(95 \% \mathrm{Cl} 3.10-8.84)$ and that of suicidal ideation was $6.20(95 \% \mathrm{Cl} 3.37-9.02)$. Since both were $>1$, this implies a positive additive interaction between sex and violence in both outcomes.

G-computation estimates of the NIE, NDE and CDE for suicidal ideation (NIE: $\beta=0.0034 ; 95 \% \mathrm{Cl} 0.0006-0.0061$; NDE: $\beta=0.0287 ; 95 \% \mathrm{Cl} 0.0245 ; 0.0328$; CDE: $\beta=0.0281$; $95 \% \mathrm{Cl} 0.0239-0.0322)$ and MDE (NIE: $\beta=0.030 ; 95 \% \mathrm{Cl}$ 0.0002-0.0058; NDE: $\beta=0.0343 ; 95 \% \mathrm{Cl} 0.0301-0.03849$; CDE: $\beta=0.0339 ; 95 \% \mathrm{Cl} 0.0298-0.0381$ ) were similar. Figure 1 shows that, in the mediation analysis, the NIE corresponded to $10.6 \%$ of the total effect of sex on MDE and $8.0 \%$ of the effect on suicidal ideation.

\section{Discussion}

We found that women are at higher risk of MDE and suicidal ideation than men, and that this association is mediated by suffering violence from a family member or acquaintance in the last 12 months. The NIE should be interpreted as the proportion of the association between sex and MDE (10.6\%) and between sex and suicidal ideation $(8.0 \%)$ that is mediated by violence. In other words, approximately $1 / 10$ of the higher risk that women have of these mental conditions is due to their increased risk of violence victimization.

Since there was no multiplicative interaction between exposure and the mediator, it was expected that the NDE would be equal to the CDE. Thus, we could also interpret the same result in terms of the mediator. If no violence had occurred in this population, $10 \%$ of the association between sex, MDE and suicidal ideation would have been prevented. However, we should point out that there was a positive additive interaction, which suggests that female violence victims would benefit more from interventions to reduce the risk of depression and suicidal ideation.

Our finding that there is a higher prevalence of MDE and suicidal ideation in women is similar to other results in the literature. ${ }^{1-4,7}$ Although biological theories of depression involve hormonal function and the performance of neurotransmitters and neuromodulators, ${ }^{22}$ social adversity, including maltreatment, gender roles, and violence, is higher in women, and its psychological impact can be very significant for both depressive disorders and suicidal ideation. $7,9,10,23$

Even when social impairment is discussed in the literature as a possible explanation for the mental health gap between men and women, most of the discussion about likely associations has focused on the fact that sex differences in depression and negative mood may be associated with biopsychosocial factors such as hormonal differences, family environment, childhood stressors and social and cultural factors. ${ }^{4,5,7,9,10,23}$

One of the most discussed (and probably the most widely accepted) hypotheses is that women's hormone levels change more throughout life due to menarche, pregnancy, menopause and contraceptive use, and this could contribute significantly to the research gap. These 
Table 1 Sample description, major depressive episodes, suicidal ideations and family violence in Brazilian adults according to sociodemographic variables, 2013 National Health Survey $(n=60,202)$

\begin{tabular}{|c|c|c|c|c|c|c|c|c|c|}
\hline \multirow[b]{2}{*}{ Variables } & \multirow{2}{*}{$\begin{array}{c}\text { Total } \\
\mathrm{n}=60,202\end{array}$} & \multirow{2}{*}{$\begin{array}{c}\text { Men } \\
\mathrm{n}=25,920\end{array}$} & \multirow{2}{*}{$\begin{array}{c}\text { Women } \\
\mathrm{n}=34,282\end{array}$} & \multicolumn{2}{|c|}{$\begin{array}{c}\text { Major depressive } \\
\text { episode }\end{array}$} & \multicolumn{2}{|c|}{ Suicidal ideations } & \multicolumn{2}{|c|}{ Violence victim } \\
\hline & & & & Men & Women & Men & Women & Men & Women \\
\hline Age & & & & $p<0.001$ & $p<0.001$ & $p=0.001$ & $p=0.022$ & $p=0.020$ & $p<0.001$ \\
\hline $18-24$ & 15.9 & 16.7 & 15.3 & 1.5 & 3.7 & 2.6 & 3.8 & 2.3 & 3.8 \\
\hline $25-34$ & 21.6 & 22.3 & 21.0 & 1.2 & 4.9 & 1.4 & 4.3 & 2.2 & 4.4 \\
\hline $35-44$ & 19.2 & 18.9 & 19.5 & 2.3 & 5.6 & 2.4 & 5.1 & 2.2 & 3.1 \\
\hline $45-54$ & 17.5 & 17.5 & 17.5 & 3.6 & 6.6 & 3.5 & 5.8 & 1.6 & 3.3 \\
\hline $55-64$ & 13.5 & 13.1 & 13.8 & 2.7 & 6.9 & 2.2 & 5.8 & 1.3 & 2.1 \\
\hline 65 or more & 12.3 & 11.4 & 13.1 & 4.5 & 6.1 & 3.4 & 4.6 & 0.9 & 1.2 \\
\hline Skin color & & & & $p=0.979$ & $p=0.606$ & $p=0.937$ & $p=0.072$ & $p=0.067$ & $p=0.001$ \\
\hline White & 47.5 & 46.8 & 48.1 & 2.4 & 5.4 & 2.4 & 4.5 & 1.5 & 2.6 \\
\hline Black & 9.2 & 9.2 & 9.2 & 2.4 & 5.7 & 2.4 & 4.9 & 2.2 & 3.9 \\
\hline Brown & 42.0 & 42.8 & 41.2 & 2.5 & 5.9 & 2.5 & 5.4 & 2.1 & 3.7 \\
\hline Yellow or indigenous & 1.4 & 1.2 & 1.5 & 2.5 & 5.3 & 3.3 & 3.6 & 0.8 & 2.0 \\
\hline Education & & & & $p<0.001$ & $\mathrm{p}<0.001$ & $p<0.001$ & $p<0.001$ & $p=0.841$ & $p<0.001$ \\
\hline $\begin{array}{l}\text { No education or } \\
\text { incomplete elementary } \\
\text { school }\end{array}$ & 21.8 & 21.1 & 22.4 & 4.1 & 8.2 & 3.9 & 6.6 & 1.7 & 2.2 \\
\hline $\begin{array}{l}\text { Complete elementary } \\
\text { school or incomplete } \\
\text { secondary school }\end{array}$ & 27.3 & 29.6 & 25.3 & 3.0 & 6.4 & 2.9 & 6.6 & 2.0 & 4.1 \\
\hline $\begin{array}{l}\text { Complete secondary } \\
\text { school or incomplete } \\
\text { higher education }\end{array}$ & 38.1 & 37.9 & 38.4 & 1.3 & 4.3 & 1.8 & 3.5 & 1.8 & 3.1 \\
\hline $\begin{array}{l}\text { Complete higher } \\
\text { education }\end{array}$ & 12.7 & 11.4 & 13.9 & 1.7 & 3.6 & 0.9 & 2.8 & 1.6 & 3.2 \\
\hline Wealth index quintiles & & & & $p=0.055$ & $p<0.001$ & $p<0.001$ & $p<0.001$ & $p<0.001$ & $p<0.001$ \\
\hline 1 (poorest) & 20.3 & 21.0 & 19.7 & 3.2 & 7.0 & 3.4 & 6.8 & 3.0 & 4.6 \\
\hline 2 & 19.8 & 19.8 & 19.8 & 2.6 & 6.5 & 2.9 & 5.9 & 1.7 & 3.7 \\
\hline 3 & 20.2 & 19.9 & 20.4 & 2.7 & 5.8 & 2.9 & 4.8 & 1.9 & 2.9 \\
\hline 4 & 20.0 & 19.6 & 20.3 & 2.2 & 4.9 & 1.9 & 4.4 & 1.2 & 2.7 \\
\hline 5 (wealthiest) & 19.8 & 19.7 & 19.8 & 1.5 & 3.8 & 1.1 & 2.6 & 1.3 & 1.9 \\
\hline
\end{tabular}

Data presented as percentages.

Table 2 Association between sex, major depressive episode, and suicidal ideation for the overall sample, stratified by violence, 2013 National Health Survey $(n=60,202)$

\begin{tabular}{lcr}
\hline Variables & Major depressive episode* & Suicidal ideation $^{\dagger}$ \\
\hline Overall sample & $2.36(2.03 ; 2.74)$ & $2.02(1.73 ; 2.36)$ \\
Non-victims & $2.24(1.91 ; 2.62)$ & $1.88(1.59 ; 2.22)$ \\
Violence victims & $3.31(2.54 ; 4.32)$ & $3.85(2.83 ; 5.25)$ \\
\hline
\end{tabular}

Data presented as odds ratio (comparing females vs. males) and $95 \%$ confidence interval.

$p$-values for interaction term in a logistic regression: * $p=0.183 ;{ }^{\dagger} p=0.050$.

changes could be directly related to mood and negative/ positive affect, and could interact with gendered intra- and interpersonal factors, such as stress. In addition, women might have a blunted cortisol response. ${ }^{10}$ Nevertheless, some research has proposed that this gap might be related to greater exposure to severe adversity, especially violence, and to structural gender inequality at macroand microlevels, despite the present lack of empirical evidence. ${ }^{5,9,10}$

In a context where suicidality is more likely a consequence of depression, these theories might be relevant, although some of the burden of suicidality is not necessarily due to depression or depressive-like symptoms. Therefore, other important predictors and mediators should be explored. ${ }^{24}$ However, in general, the reasons women are more affected than men by depression and/or suicidality remain unresolved in the literature, and biological factors do not seem to fully explain it.

Violence against women can take many forms, such as physical, sexual and emotional/psychological, and these forms can coexist. ${ }^{14,25,26}$ Several studies have shown female violence victims are at an increased risk of mental disorders. ${ }^{25-29}$ Furthermore, women who have experienced repeated domestic violence and abuse, other forms of violent victimization, or direct expressions of rage or aggression have a strong tendency to suppress their emotions. ${ }^{5}$ This could be related to other behaviors, such as substance use and other psychological consequences of violence, including suicidal ideation. . $^{26,27,29,30}$ When combined with the submissive nature of some women, 
continuous exposure to stressful events, such as violence, could lead to extreme vulnerability, fear and feelings of inability or powerlessness, which could cause or contribute to mental health problems.

Sociocultural factors, such as the stress associated with traditional female roles, can affect women's mental health. $5,7,8,10$ In this study we found important associations between sociodemographic characteristics and depression and suicidal ideation in women, a relationship that has been demonstrated in other studies. ${ }^{1,4,31}$ For the men in our sample, no associations were found between skin color and either outcome, between education and violence victimization, or between WIQ and MDE. In Brazil, skin color, education and WIQ are markers of social vulnerability, which can be associated with mental health conditions. $^{32,33}$ The fact that important social determinants were associated with outcomes for Brazilian women but not for men could indicate that these determinants are among the sociocultural markers that affect women's mental health. We also found that women are more likely than men to suffer violence by a family member or acquaintance, which has also been reported in previous studies. ${ }^{11,12,15}$ However, these findings also indicate the importance of investigating these mental health conditions and their greater occurrence among women from a social determinants of health perspective.

It is important to consider some limitations of our study. First, since we did not have access to all the necessary information on losses and refusals, we could not assess their impact on our measures of association. However, the losses were relatively small, and they do not seem related to exposure, since the prevalence of females and depression/suicide were similar to that reported in other studies. $^{2}$ The PHQ-9 uses an algorithm to assess depression that reproduces DSM-IV diagnostic criteria. Nevertheless, as with any screening test, classification errors could have occurred, since a clinical interview was not conducted for diagnostic confirmation. Similarly, suicidal ideation and violence were self-reported and evaluated with a single question. Thus, underreporting could have also been an issue in this study. However, we considered that if there was a classification error for any of these cases it would be non-differential, thus the measures of association reported in this study could be underestimated. Such underestimation could influence our findings, leading to underestimation of the mediating effect of violence, for example. In addition, although longitudinal measures would be preferable for assessing the mediating effect of violence, we find it more plausible to think that depression and suicidal ideation are a consequence of violence, at least in this case, and not the other way around, and there is evidence of such a longitudinal association in the literature. ${ }^{12,13}$ In addition, respondents reported the occurrence of violence in the last 12 months, but depression and suicidal ideation in the last two weeks.

We only studied violence from family members or acquaintances, which might have limited our capacity to determine which kind of violence would be more important in the explored mediators. However, the available data was inappropriate for answering this specific question, since the type of violence was self-reported and the occurrence of violence was likely underestimated. Thus, the answer to this question should be explored in other studies with higher specificity.

On the other hand, we used a representative sample of the entire Brazilian population that included more than 60,000 individuals. We also assessed mediation with a novel method, using a counterfactual framework that avoided overadjustment and extracted measures that could be considered confounders. Since violence and mental health outcomes could both be consequences of sociodemographic characteristics, to correctly calculate NDE and NIE, we had to adjust our models for those variables, and given that adjusting for sociodemographic characteristics in a regular regression model would have been inadequate (leading to overadjustment), they had to be treated as post-confounders. Thus, estimating from G-computation is more adequate for evaluating the mediating effect of violence while adjusting for sociodemographic variables. ${ }^{21}$

Finally, our study has shown evidence that violence is a mediator in the causal pathway between sex, and depression and suicidal ideation. Avoiding any kind of violence is already considered an important objective worldwide, and it should be pursued independently of any association with mental health. However, we find it relevant to highlight the importance of psychosocial issues, especially violence from family members or acquaintances, in the etiology of mental health conditions, since they partially explain why women are more affected than men. Our results indicate that one likely reason women have a higher burden of depression and suicidality is the violence they suffer, i.e., vulnerability to these mental health problems is not as much an inherent condition as a consequence of external psychosocial factors. Therefore, it is important to consider these issues in the planning and implementation of public mental health policies, as well as in more specific clinical approaches, especially those regarding women's mental health.

\section{Acknowledgements}

This study was conducted in a graduate program supported by the Coordenação de Aperfeiçoamento de Pessoal de Nível Superior (CAPES; Finance Code 001).

\section{Disclosure}

The authors report no conflicts of interest.

\section{References}

1 Bromet E, Andrade LH, Hwang I, Sampson NA, Alonso J, de Girolamo G, et al. Cross-national epidemiology of DSM-IV major depressive episode. BMC Med. 2011;9:90.

2 Ferrari AJ, Somerville AJ, Baxter AJ, Norman R, Patten SB, Vos T, et al. Global variation in the prevalence and incidence of major depressive disorder: a systematic review of the epidemiological literature. Psychol Med. 2013;43:471-81.

3 Ferrari AJ, Charlson FJ, Norman RE, Patten SB, Freedman G, Murray $\mathrm{CJ}$, et al. Burden of depressive disorders by country, sex, age, and year: findings from the global burden of disease study 2010 . PLoS Med. 2013;10:e1001547.

4 Kessler RC, Bromet EJ. The epidemiology of depression across cultures. Annu Rev Public Health. 2013;34:119-38. 
5 Maji S. Society and 'good woman': a critical review of gender difference in depression. Int J Soc Psychiatry. 2018;64:396-405.

6 Girgus JS, Yang K, Ferri CV. The gender difference in depression: are elderly women at greater risk for depression than elderly men? Geriatrics (Basel). 2017;2(4).

7 Straiton ML, Roen K, Hjelmeland H. Gender roles, suicidal ideation, and self-harming in young adults. Arch Suicide Res. 2012;16:29-43.

8 Bhui K. Gender, power and mental illness. Br J Psychiatry. 2018; 212:191-2.

9 Howard LM, Ehrlich AM, Gamlen F, Oram S. Gender-neutral mental health research is sex and gender biased. Lancet Psychiatry. 2017;4:9-11.

10 Kuehner $\mathrm{C}$. Why is depression more common among women than among men? Lancet Psychiatry. 2017;4:146-58.

11 Bhui K. Violence, trauma, testimonials and truth. $\mathrm{Br} \mathrm{J}$ Psychiatry. 2018;213:625-6.

12 Gallo EA, De Mola CL, Wehrmeister F, Gonçalves H, Kieling C, Murray J. Childhood maltreatment preceding depressive disorder at age 18 years: a prospective Brazilian birth cohort study. J Affect Disord. 2017;217:218-24.

13 Gallo EA, Munhoz TN, de Mola CL, Murray J. Gender differences in the effects of childhood maltreatment on adult depression and anxiety: a systematic review and meta-analysis. Child Abus Negl. 2018; 79:107-14.

14 Garcia-Moreno C, Zimmerman C, Morris-Gehring A, Heise L, Amin A, Abrahams $\mathrm{N}$, et al. Addressing violence against women: a call to action. Lancet. 2015;385:1685-95.

15 Wilson NL. Violence against female adolescents in low- and middleincome countries: evidence from 36 national household surveys. J Interpers Violence. 2018 Aug 27:886260518792971. doi: http:// $10.1177 / 0886260518792971$. Online ahead of print.

16 United Nations (UN). The sustainable development goals report [Internet]. 2019 [cited 2019 Oct 29]. http://unstats.un.org/sdgs/report/ 2019/The-Sustainable-Development-Goals-Report-2019.pdf

17 GBD 2017 DALYs and HALE Collaborators. Global, regional, and national disability-adjusted life-years (DALYs) for 359 diseases and injuries and healthy life expectancy (HALE) for 195 countries and territories, 1990-2017: a systematic analysis for the global burden of disease study 2017. Lancet. 2018;392:1859-922.

18 Instituto Brasileiro de Geografia e Estatística. População [Internet] 2019. [cited 2019 Oct 29]. https://ww2.ibge.gov.br/home/estatistica/ populacao/pns/2013/

19 Santos IS, Tavares BF, Munhoz TN, Almeida LS, da Silva NT, Tams BD, et al. [Sensitivity and specificity of the Patient Health Questionnaire-9
(PHQ-9) among adults from the general population]. Cad Saude Publica. 2013;29:1533-43.

20 VanderWeele TJ, Knol MJ. A tutorial on interaction. Epidemiol Methods. 2014;3:33-72.

21 Richiardi L, Bellocco R, Zugna D. Mediation analysis in epidemiology: methods, interpretation and bias. Int J Epidemiol. 2013;42:1511-9.

22 Otte C, Gold SM, Penninx BW, Pariante CM, Etkin A, Fava M, et al. Major depressive disorder. Nat Rev Dis Primers. 2016;2:16065.

23 Rhodes AE, Boyle MH, Bridge JA, Sinyor M, Links PS, Tonmyr L, et al. Antecedents and sex/gender differences in youth suicidal behavior. World J Psychiatry. 2014;4:120-32.

24 Morales-Vives F, Dueñas JM. Predicting suicidal ideation in adolescent boys and girls: the role of psychological maturity, personality traits, depression and life satisfaction. Span J Psychol. 2018;21: E10.

25 da Silva LE, de Oliveira ML. Violence against women: systematic review of the Brazilian scientific literature within the period from 2009 to 2013. Cien Saude Colet. 2015;20:3523-32.

26 Stewart DE, Vigod SN. Update on mental health aspects of intimate partner violence. Med Clin North Am. 2019;103:735-49.

27 Oram S, Khalifeh H, Howard LM. Violence against women and mental health. Lancet Psychiatry. 2017;4:159-70.

28 Beydoun HA, Beydoun MA, Kaufman JS, Lo B, Zonderman AB. Intimate partner violence against adult women and its association with major depressive disorder, depressive symptoms and postpartum depression: a systematic review and meta-analysis. Soc Sci Med. 2012;75:959-75.

29 Devries KM, Mak JY, Bacchus LJ, Child JC, Falder G, Petzold M, et al. Intimate partner violence and incident depressive symptoms and suicide attempts: a systematic review of longitudinal studies. PLoS Med. 2013;10:e1001439.

30 Beydoun HA, Williams M, Beydoun MA, Eid SM, Zonderman AB. Relationship of physical intimate partner violence with mental health diagnoses in the nationwide emergency department sample. J Womens Health (Larchmt). 2017;26:141-51.

31 Munhoz TN, Nunes BP, Wehrmeister FC, Santos IS, Matijasevich A. A nationwide population-based study of depression in Brazil. J Affect Disord. 2016;192:226-33.

32 Almeida-Filho N, Lessa I, Magalhães L, Araujo MJ, Aquino E, James SA, et al. Social inequality and depressive disorders in Bahia, Brazil: interactions of gender, ethnicity, and social class. Soc Sci Med. 2004; 59:1339-53.

33 Antunes JL. [Socioeconomic status and health: a discussion of two paradigms]. Rev Saude Publica. 2008;42:562-7. 